\title{
Using Medicare Data to Assess the Proarrhythmic Risk of Non-Cardiac Treatment Drugs that Prolong the QT Interval in Older Adults: An Observational Cohort Study
}

\author{
Kin Wah Fung ${ }^{1} \mathbb{D} \cdot$ Fitsum Baye $^{1} \cdot$ Joan Kapusnik-Uner ${ }^{2,3} \cdot$ Clement J. McDonald $^{1}$
}

Accepted: 19 January 2021 / Published online: 10 February 2021

(c) The Author(s) 2021

\begin{abstract}
Introduction Serious cardiac arrhythmias caused by QT-prolonging drugs are difficult to predict based on physiological measurement and pre-approval clinical trials. Post-marketing surveillance and monitoring are important to generate safety data.

Objectives To assess whether an observational study using Medicare claims data can detect the arrhythmogenic risk of QT-prolonging drugs.

Methods We identified 17 QT-prolonging drugs with known risk of torsades des pointes (TdP) that were not used to treat cardiac arrhythmias. Amoxicillin and four serotonin-norepinephrine reuptake inhibitors (SNRIs) were used as controls. De-identified claims data of 1.2 million Medicare beneficiaries were accessed. Two separate Cox regressions were done for short-term and chronic-use drugs. The primary outcome was a composite of ventricular arrhythmias and/or sudden death, identified by ICD diagnostic codes. We explored the independent effect of each study drug on the outcomes. Other covariates included patient demographics, comorbidities, and known risk factors for drug-induced cardiac arrhythmia.

Results We were able to detect increased risk in 14 of 17 study drugs (82.3\%), and none of the control drugs. Among the fluoroquinolones, ciprofloxacin was the safest. Azithromycin and clarithromycin were relatively safe compared to erythromycin. Compared to SNRIs, both citalopram and escitalopram had increased risk, more so with escitalopram than citalopram. Comorbidities associated with increased risk included ischemic heart disease, electrolyte imbalance, bradycardia, acute myocardial infarction, heart failure, and chronic kidney and liver disease.

Conclusion Medicare data can be utilized for post-marketing surveillance and monitoring of the proarrhythmic risk of QTprolonging drugs in older adults.
\end{abstract}

Kin Wah Fung

kfung@mail.nih.gov

1 Lister Hill National Center for Biomedical Communications, National Library of Medicine, U.S. National Institutes of Health, 8600 Rockville Pike, Bethesda, MD 20894, USA

2 First Databank. Inc., San Francisco, CA, USA

3 University of California, San Francisco, CA, USA

\section{Key Points}

The Medicare claims database can be a useful resource in monitoring serious cardiac adverse effects of QTprolonging drugs, which are generally difficult to predict and quantify.

Based on data from 1.2 million Medicare beneficiaries and over 5 million patient-years of follow-up, we detected an increased risk of some commonly prescribed drugs including levofloxacin, citalopram, and escitalopram for ventricular arrhythmia and sudden death. 


\section{Introduction}

The association of symptomatic cardiac arrhythmia with QT-prolonging drugs can be traced back to the first report of syncope in patients receiving quinidine, almost a hundred years ago [1]. It was later found that these dramatic episodes of syncope were often associated with a specific pattern of ventricular tachycardia called torsades des pointes (TdP) [2]. As most of the early cases of TdP were observed in patients treated for cardiac arrhythmia, it was assumed that this rare form of arrhythmia was mainly related to antiarrhythmic treatment drugs. However, the attitude towards TdP changed radically when it was discovered that common, non-cardiac treatment drugs taken by millions of patients could also cause QT prolongation and TdP. This led to tight scrutiny and eventual withdrawal from the market of some drugs such as terfenadine, astemizole, and cisapride [3]. In the 1990s, the US Food and Drug Administration (FDA) and the European Medicine Agency (EMA) began requiring preclinical and clinical tests for the QT-prolonging potential of developing drugs $[4,5]$. Since 1989 , at least 14 clinically important drugs have been withdrawn from the market due to TdP, with an unknown number of developing drugs halted due to the same concern [6]. Over the past two decades, QT prolongation has become one of the most common reasons for drug withdrawal or relabeling [7]. Recently, in the midst of the COVID-19 (Coronavirus Disease 2019) pandemic, the enthusiasm over the use of hydroxychloroquine and azithromycin as potential prophylaxis or treatment has raised concerns because both drugs have been known to cause TdP [8]. After issuing an emergency use authorization for hydroxychloroquine and chloroquine for treatment of hospitalized COVID-19 patients, the FDA had to issue a drug safety communication recommending against the use of these drugs outside of the hospital or clinical trial settings due to inability to monitor for their potential increased risk for ventricular tachycardia [9]. Subsequently, clinical reports have documented occurrences of significant QT prolongation and TdP in COVID-19 patients receiving these drugs which, together with the lack of evidence of therapeutic efficacy, led to revocation of the emergency use authorization by the FDA [10-13].

Despite stringent pre-marketing requirement for testing, physiologic measurement of QT prolongation is an unreliable predictor of the likelihood of ventricular arrhythmia [14-16]. Not all QT-prolonging drugs are proarrhythmic and the absence of QT prolongation is not a guarantee for safety. In vitro measurement of QT prolongation of a drug often does not correlate well with the clinical incidence of arrhythmia. Apart from the pharmacodynamics of the concerned drug, the likelihood of arrhythmia also depends on patient factors such as genetic disposition (e.g., female gender, congenital long QT syndrome), co-morbidities (e.g., hypokalemia, bradycardia, structural heart disease) and interaction with other drugs (e.g., additive effects of other QT prolongers or pharmacokinetic interactions that increase drug concentrations, putting patients at increased risk of QT prolongation) [17-21]. Since it is not possible to precisely measure the risk that a drug will prolong the QT interval in an individual patient and thus increase the risk for TdP, the most reliable predictor of safety for any QT-prolonging drug is its history of safe administration across a large number of patients [22]. Therefore, outcome studies based on large clinical data sets are especially important to assess the risk of drug-induced arrhythmia [23].

The risk of specific drugs to cause TdP is difficult to quantify because these events are generally rare and their transient nature makes them hard to diagnose. One study estimated the overall incidence of drug-induced $\mathrm{TdP}$ to be 2.5 and 4.0 per million per year for males and females, respectively [24]. It follows that drug-induced arrhythmia studies based on general population data will require millions of patients with multiyear follow-up to have enough statistical power. The claims data from the US Centers for Medicare and Medicaid Services (CMS) have been made available for such research [25]. The main strength of the Medicare data is population coverage. It is estimated that over $93 \%$ of adults age 65 years and over are enrolled in Medicare, making Medicare data one of the richest sources of healthcare utilization information in the country [26, 27]. Currently, there are over 45 million Medicare beneficiaries. The data available to researchers include patient demographics, diagnoses, procedures, medications, and service encounter dates. Data are generally made available within a year. Patient-level data can be linked across years. We present a study using the CMS data to assess the risk of sudden death and ventricular arrhythmias for a subset of commonly prescribed non-cardiac treatment QT-prolonging drugs known to be associated with TdP. We postulate that an observation study using CMS data is able to emulate clinical studies in detecting the increased risks of known drugs and potentiating co-morbidities. New insights can also be gained regarding the relative risks of drugs within drug classes. To the best of our knowledge, this is the first study using the CMS data set to address this particular problem. We present our findings and discuss potential applications of this approach in postmarketing surveillance. 


\section{Materials and Methods}

\subsection{Study Population}

Our study population came from a $20 \%$ random sample of the Medicare beneficiaries who first enrolled in Medicare after 1 January 2007 (the first full year of prescription drug-Part D coverage) and after reaching the age of 65 years (Fig. 1). Individuals became eligible for the study at their Part D entitlement. In this observational cohort study, we followed patients from their Part D enrollment (accounting for left truncation [28]) to their first record of ventricular arrhythmias (VA), sudden death (SD), death, switch to a capitated plan, disenrollment from Medicare, or end of 2016, whichever came first. To ensure that all patients have been followed up for a meaningful period, we excluded patients with less than 6 months of follow-up. To ensure we have full inpatient and outpatient claims data, we censored patients when they dis-enrolled from Parts A (hospital insurances) and B (medical insurances). We used 10 years of de-identified Medicare claims data from 1 January 2007 through 31 December 2016 from the CMS
VRDC (Virtual Research Data Center) system. This study was exempted from human subject research review by the Office of Human Subjects Research Protections, National Institutes of Health.

\subsection{Drugs, Controls, and Exposure Definition}

We identified select drugs known to have a risk of TdP based on a list obtained from the CredibleMeds online resource in February 2019. To date, all of drugs in this study are still on the list $[15,29]$. We chose from drugs that had not been withdrawn from the US market. These drugs were extracted using generic names from Part D prescription claims data. We excluded the antiarrhythmic drugs from our analysis because one of our outcome events, ventricular arrhythmia, might be the indication for which these drugs were prescribed. We excluded drugs or drug classes that were taken by less than $1 \%$ of the study population because of the lack of statistical power. Of the 48 drugs with known TdP risk, ten had been withdrawn from the US market, nine were antiarrhythmics and 12 had low usage. The full list of drugs can be found in the Online Supplementary Material (OSM), S1. For the remaining 17 drugs, we did separate
Fig. 1 Schematic diagram of cohort and analysis

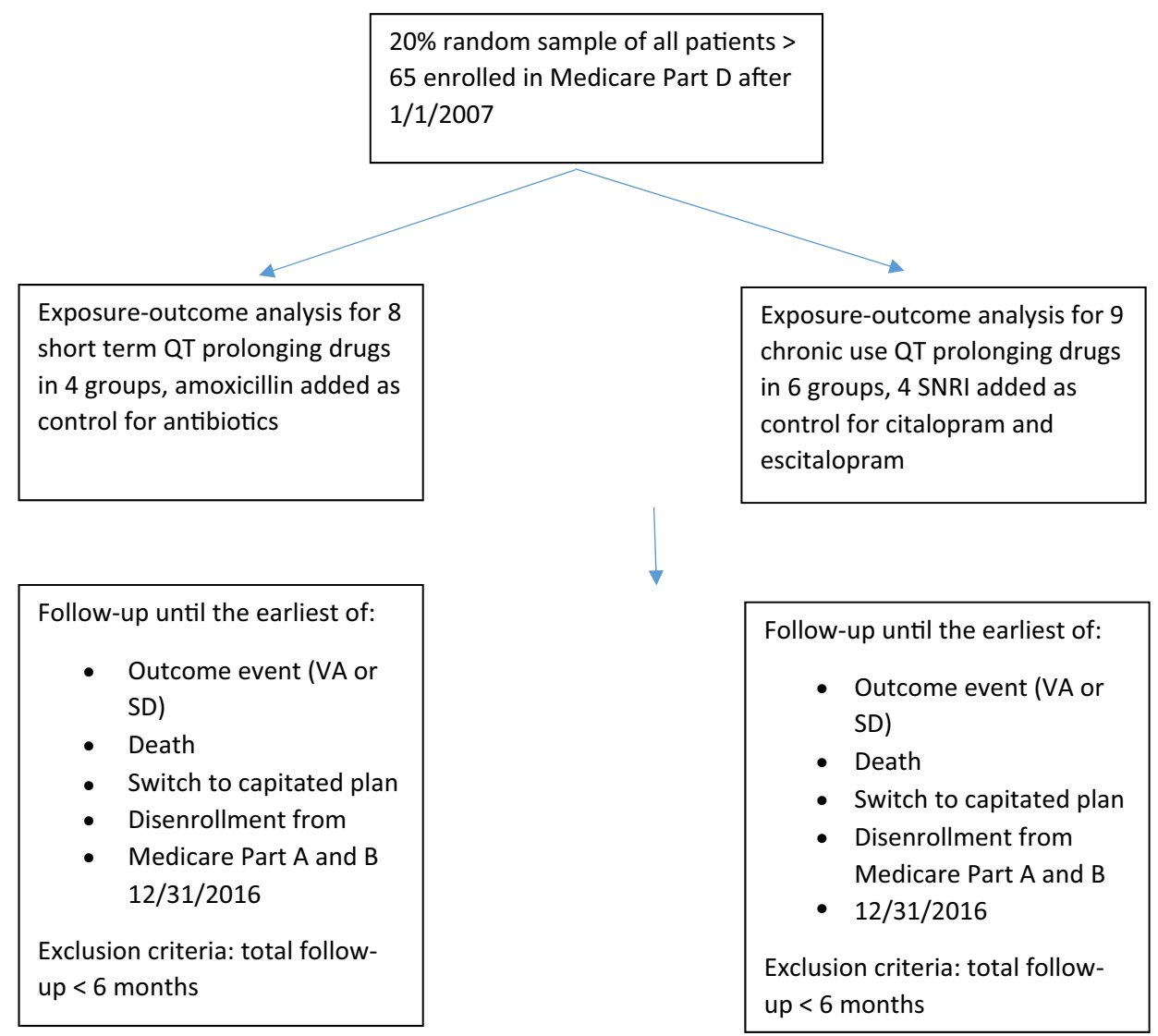


analyses for short-term versus chronic-usage drugs because of their different prescription patterns, risk profiles, and possible cumulative effects. We used 30 days in the median days of supply (OSM, S2) as the cut-off to distinguish between short-term and chronic-usage drugs. We studied eight short-term drugs in one analysis-three fluoroquinolone antibacterials (ciprofloxacin, levofloxacin, and moxifloxacin, analyzed individually), three macrolide antibacterials (azithromycin, clarithromycin, and erythromycin, analyzed individually), one antifungal (fluconazole), and one antiemetic (ondansetron). We added amoxicillin as a control for the antibacterials. We studied nine chronic usage drugs in a separate analysis - two selective serotonin reuptake inhibitor (SSRI) antidepressants (citalopram and escitalopram, analyzed individually), three antipsychotics (haloperidol, thioridazine, and chlorpromazine, analyzed as a group because of the small number of patients), two phosphodiesterase inhibitors (PDEIs; cilostazol and anagrelide, analyzed as a group because of the small number of patients), one anti-rheumatic drug (hydroxychloroquine), and one anti-Alzheimer drug (donepezil). We added four serotonin-norepinephrine reuptake inhibitor (SNRI) antidepressants that did not have a known risk of TdP as control for the SSRI antidepressants. We matched the selected drugs to the CMS data using their generic drug names. We only included systemic dose forms (e.g., tablet, capsule, vial) and excluded topical and nonsystemic dose forms (e.g., ointment, eye drop).

We considered that patients were using a study drug from the date of dispensing to the end of supply date. To compensate for drug stockpiling (i.e., patients may still be on a drug even though they have apparently run out, because they have accumulated excess supply), we added an extra 10 days for short-term drugs and 30 days for chronic-use drugs after the end of supply date. The extra days represented the median days of supply for most short-term and chronic-use drugs, respectively. A patient's drug use status was constantly updated throughout the follow-up period whenever an outcome event occurred. Drug-usage status was categorized into three mutually exclusive categories as current, former, and never users based on the time since last day of supply. For short-term drugs, a patient's usage status was defined as current user from the prescription day to 10 days after the last day of supply. If the prescription was not renewed, the patient would become a former user. Never users were patients who never had a prescription for the drug. For chronic-use drugs, current usage covered the period from prescription date to within 30 days of the last day of supply. For chronic-usage drugs, in order to study the possible cumulative effect, we further divided current users into two sub-groups: patients with $>12$ months or $\leq$ 12 months of prior cumulative usage. The median days of supply and daily doses of study and control drugs are available as OSM, S3.

\subsection{Outcome Events and Co-Morbidities}

Our primary outcome was a composite of ventricular arrhythmias (VAs) and/or sudden death (SD). These events were identified by diagnosis codes-ICD-9-CM (before October 2015) and ICD-10-CM (after October 2015), regardless of whether they were used as the principal or secondary diagnoses. The respective ICD-9-CM/ICD10-CM codes were-VA: Paroxysmal ventricular tachycardia (427.1/I47.2), Ventricular fibrillation (427.41/I49.01) and Ventricular flutter (427.42/I49.02); SD: Cardiac arrest (427.5/I46.9) and Instantaneous death (798.1, no corresponding code in ICD-10-CM). Since the conditions leading to cardiac arrest and instantaneous death were heterogeneous and could include non-cardiac causes, we did a secondary analysis with VA alone as outcome.

To account for the effect of co-morbidities, we used the CMS Chronic Conditions Warehouse (CCW) co-morbidity flags to identify 46 chronic conditions with $>1 \%$ prevalence. Some of these co-morbidities were known to be risk factors for drug-induced TdP (e.g., heart failure, liver and kidney diseases). We added two other risk factors not covered by the CCW flags-electrolyte imbalance (including hypokalemia) and bradycardia, identified by ICD codes. We did not include congenital long QT syndrome as a covariate in our analysis because of the low incidence ( $0.4 \%$ of patients).

\subsection{Statistical Analysis}

For our primary analysis, we used two separate Cox regression models to explore the independent effect of short-term and chronic-usage drugs on the outcomes. To account for demographic, socioeconomic, and geographic variations, we included these patient characteristics as co-variates-gender, race, degree of low-income subsidy (as a surrogate for income), rural residence indicator, and year of Part D entry (as a surrogate for age, since most patients enrolled at 65 years of age). We also included all the $\mathrm{CCW}$ co-morbidity flags described above as proxies for a patient's medical burdens and TdP risk. To minimize the immortal time bias we treated all drug usage and patient characteristics (except gender, race, and year of Part D entry) as time-varying covariates $[30,31]$. This means that drug-usage status is determined repeatedly at each event time throughout the analysis, based on prescription history at that time. At any point in time, a patient can be only one of current, former, or past user for a particular drug, but their usage status will change with time. We did not consider drug dosage as a covariate because of sample-size limitation. To compensate for the imbalance in covariate distribution among drug groups, we used a propensity score (PS) weighting method. We derived two sets of PS weightings for short-term and chronic drugusage patterns. Creating all possible combinations of drugs 
would significantly reduce the sample size in each group. We decided on four mutually exclusive treatment groups for short term drugs: (1) only antibiotics, (2) only nonantibiotics, (3) both antibiotics and non-antibiotics, and (4) none. Then we estimated PS for the treatment groups based on patient demographics and co-morbidities, using a multinomial logistic regression [32]. Similarly, we used seven mutually exclusive groups for the chronic-usage drugs: (1) antidepressants only, (2) antipsychotics only, (3) PDE3 inhibitors only, (4) hydroxychloroquine only, (5) donepezil only, (6) any combination of the five groups, and (7) none. Patient characteristics before and after PS adjustment are available as OSM, S4, S5a, and S5b. Cox regression analyses were weighted by the inverse propensity score [33]. To assess the impact of possible immortal time bias due to the exclusion of patients with less than 6 months' follow-up, we did a sensitivity analysis without this exclusion. All analyses were performed using SAS Enterprise Guide software.

\section{Results}

\subsection{Study Population}

From our 20\% random sample of Medicare enrollees, $1,265,921$ patients satisfied all our selection criteria (Table 1). Starting with their part D enrollment, these patients had a median follow-up of 3.7 years, giving a total of 5,120,909 patient-years. Follow-up ended when they developed an outcome event (3.1\%), died (4.2\%), switched to a capitated plan (13.9\%), or reached the end of our study (78.9\%). The proportion of female was $58.5 \%$. Non-Hispanic White and African American made up 81.4\% and 7.2\%, respectively. About $19 \%$ of patients were of low income, either dually enrolled to Medicare and Medicaid, or receiving a low-income subsidy. Rural residents made up $22.8 \%$. Among the short-term study drugs, azithromycin was the most common and used by $35.3 \%$ of patients. The control drug amoxicillin was used by $40 \%$. Among the chronic usage study drugs, citalopram was the most common $(6.1 \%)$ followed by escitalopram (5.1\%). The SNRI antidepressant control drugs were used by $6.8 \%$ of patients. The three most commonly observed proarrhythmic co-morbidities were ischemic heart disease (28.3\%), hypothyroidism (20.7\%), and chronic kidney disease (19.1\%).

\subsection{Short-Term Drugs}

Among the fluoroquinolones, levofloxacin and moxifloxacin both showed increased risks for the primary outcome, i.e., VA and/or SD. Levofloxacin use increased the risk by $51 \%$ (hazard ratio $(\mathrm{HR})=1.51 ; 95 \%$ confidence interval (CI) 1.44-1.57) compared to never users, 63\% (HR 1.63;
95\% CI 1.56-1.70) compared to former users, and 51\% (HR $1.51 ; 95 \%$ CI 1.42-1.61) compared to amoxicillin controls (Table 2). Moxifloxacin use increased the risk by 23\% (HR 1.23 ; 95\% CI 1.03-1.45) compared to never users, 35\% (HR $1.35 ; 95 \%$ CI $1.13-1.60$ ) compared to former users, and $23 \%$ (HR 1.23; 95\% CI 1.03-1.46) compared to amoxicillin controls. Ciprofloxacin use increased risk by $30 \%$ (HR 1.30; 95\% CI 1.24-1.36) compared to former users, but no difference from amoxicillin controls or never users.

Among the macrolides, erythromycin had increased risk of $63 \%$ (HR 1.63; 95\% CI 1.32-2.02) compared to never users, 95\% (HR 1.95; 95\% CI 1.57-2.43) compared to former users, and $63 \%$ (HR 1.63; 95\% CI 1.23-2.03) compared to amoxicillin controls. The only significant result for azithromycin user was an increased risk of $22 \%$ (HR 1.22; 95\% CI 1.16-1.28) when compared to former users. For clarithromycin, there was a $21 \%$ (HR $0.79 ; 95 \%$ CI $0.66-0.95$ ) reduced risks compared to never users and a $21 \%$ (HR $0.79 ; 95 \% \mathrm{CI}$ 0.66-0.96) reduced risk compared to amoxicillin controls, and no significant difference from former users.

For the control antibiotic amoxicillin, current users was not associated with increased or decreased risk when compared to never users. There was an increased risk of $24 \%$ (HR 1.24; 95\% CI 1.19-1.29) compared with former users.

Both fluconazole and ondansetron showed increased risks. Fluconazole use increased the risk by $123 \%$ (HR 2.23; 95\% CI 2.15-2.32) compared to never users and 58\% (HR 1.58 ; $95 \%$ CI $1.53-1.65$ ) compared to former users. Ondansetron use increased the risk by $205 \%$ (HR 3.05; 95\% CI 2.96-3.14) compared to never users and 77\% (HR 1.77; 95\% CI 1.72-1.83) compared to former users.

As for demographic factors, being female reduced the risk by $30 \%$ (HR 0.70; 95\% CI 0.69-0.70, see Sect. 4 for interpretation in relation to drug usage). Younger patients (newer enrollment to Part D) had reduced risk. The risk was increased for African Americans by 13\% (HR 1.13; 95\% CI 1.11-1.14), while other races had decreased risks compared to Whites. Lower income (either dually enrolled for Medicare and Medicare or on low-income subsidy) was associated with increased risk while rural residence had a small protective effect. All the known proarrhythmic co-morbidities (except hypothyroidism) were associated with increased risks. Among other commonly occurring co-morbidities that were not specifically identified as proarrhythmic, hypertension, anemia, diabetes, depression, and obesity were associated with increased risks.

For the secondary outcome (i.e., VA only), the trends generally mirrored the primary outcome (i.e., VA and $\mathrm{SD})$. The exceptions were that the following comparisons became non-significant: moxifloxacin current versus never user, clarithromycin versus amoxicillin, African American versus White, and other ethnicity versus White. Three comparisons that showed no effect on VA and SD 
Table 1 Study population, drugs, and co-morbidities

\begin{tabular}{|c|c|}
\hline & No. of patients (\%) \\
\hline Total & $1,265,921(100)$ \\
\hline \multicolumn{2}{|l|}{ Follow-up ended at } \\
\hline 1. Ventricular arrhythmias (VA) and/or sudden death (SD) & $38,911(3.1)$ \\
\hline 2. Death & $52,979(4.2)$ \\
\hline 3. Switch to capitated plan & $175,829(13.9)$ \\
\hline 4. Disenrollment from Medicare or end of study & $994,202(78.9)$ \\
\hline \multicolumn{2}{|c|}{$\begin{array}{l}* * \text { Median length of follow-up } 3.7 \text { years, total } 5,120,909 \text { patient-years, median age at part D entry } 65.0 \\
\text { years, median age at end of follow-up } 69.4 \text { years }\end{array}$} \\
\hline \multicolumn{2}{|l|}{ Demographics } \\
\hline Female & $741,024(58.5)$ \\
\hline White & $1,029,962(81.4)$ \\
\hline African American & $90,865(7.2)$ \\
\hline Hispanic & $69,848(5.5)$ \\
\hline Asian & $32,152(2.5)$ \\
\hline Other & $43,094(3.4)$ \\
\hline Dual Medicare and Medicaid & $207,021(16.4)$ \\
\hline Non-dual, on low-income subsidy & $32,776(2.6)$ \\
\hline Rural residence & $288,307(22.8)$ \\
\hline \multicolumn{2}{|l|}{ Drug exposure—short-term drugs } \\
\hline Ciprofloxacin & $343,320(27.1)$ \\
\hline Levofloxacin & $239,083(18.9)$ \\
\hline Moxifloxacin & $26,528(2.1)$ \\
\hline Azithromycin & $446,943(35.3)$ \\
\hline Clarithromycin & $54,805(4.3)$ \\
\hline Erythromycin & $11,695(0.9)$ \\
\hline Fluconazole & $116,539(9.2)$ \\
\hline Ondansetron & $116,149(9.2)$ \\
\hline Amoxicillin (control) & $505,805(40)$ \\
\hline \multicolumn{2}{|l|}{ Drug exposure—chronic-usage drugs } \\
\hline Citalopram & $77,558(6.1)$ \\
\hline Escitalopram & $65,142(5.1)$ \\
\hline Antipsychotics & $5,359(0.4)$ \\
\hline Phosphodiesterase (PDE3) inhibitors & $7,711(0.6)$ \\
\hline Hydroxychloroquine & $17,201(1.4)$ \\
\hline Donepezil & $19,853(1.6)$ \\
\hline SNRI antidepressants (control) & $86,098(6.8)$ \\
\hline \multicolumn{2}{|l|}{ Proarrhythmic risk factors } \\
\hline Ischemic heart disease & $358,876(28.3)$ \\
\hline Hypothyroidism & $262,066(20.7)$ \\
\hline Chronic kidney disease & $241,813(19.1)$ \\
\hline Bradycardia & $173,661(13.7)$ \\
\hline Electrolyte imbalance (including hypokalemia) & $156,606(12.4)$ \\
\hline Heart failure & $142,555(11.3)$ \\
\hline Liver disease and cirrhosis & $85,854(6.8)$ \\
\hline Acute myocardial infarction & $25,561(2.0)$ \\
\hline
\end{tabular}

showed reduced risk for VA only: ciprofloxacin current versus never user, amoxicillin current versus never user, and clarithromycin current versus former user. The sensitivity analysis without exclusion of patients with $<$ 6 months' follow-up showed no significant change in the results. 
Table 2 Results for short-term drugs

\begin{tabular}{|c|c|c|c|}
\hline Treatment category & Reference group & $\begin{array}{l}\text { VA and/or SD } \\
\text { HR }(95 \% \mathrm{CI})\end{array}$ & $\begin{array}{l}\text { VA } \\
\text { HR }(95 \% \mathrm{CI})\end{array}$ \\
\hline \multicolumn{4}{|l|}{ Current vs. never user } \\
\hline Ciprofloxacin current user & Never user & $1.00(0.95-1.05)$ & $0.93(0.87-0.99)$ \\
\hline Levofloxacin current user & Never user & $1.51(1.44-1.57)$ & $1.28(1.20-1.36)$ \\
\hline Moxifloxacin current user & Never user & $1.23(1.03-1.45)$ & $1.18(0.95-1.47)$ \\
\hline Azithromycin current user & Never user & $0.98(0.93-1.03)$ & $0.99(0.93-1.05)$ \\
\hline Clarithromycin current user & Never user & $0.79(0.66-0.95)$ & $0.73(0.58-0.92)$ \\
\hline Erythromycin current user & Never user & $1.63(1.32-2.02)$ & $1.83(1.42-2.36)$ \\
\hline Amoxicillin (control) current user & Never user & $1.00(0.96-1.04)$ & $0.93(0.88-0.98)$ \\
\hline Fluconazole current user & Never user & $2.23(2.15-2.32)$ & $2.07(1.97-2.17)$ \\
\hline Ondansetron current user & Never user & $3.05(2.96-3.14)$ & $2.99(2.88-3.10)$ \\
\hline \multicolumn{4}{|l|}{ Antibiotics vs. control } \\
\hline Ciprofloxacin current user & Amoxicillin current user & $1.00(0.94-1.07)$ & $1.00(0.92-1.09)$ \\
\hline Levofloxacin current user & Amoxicillin current user & $1.51(1.42-1.61)$ & $1.38(1.27-1.50)$ \\
\hline Moxifloxacin current user & Amoxicillin current user & $1.23(1.03-1.46)$ & $1.28(1.02-1.60)$ \\
\hline Azithromycin current user & Amoxicillin current user & $0.98(0.92-1.05)$ & $1.07(0.98-1.16)$ \\
\hline Clarithromycin current user & Amoxicillin current user & $0.79(0.66-0.96)$ & $0.79(0.62-1.00)$ \\
\hline Erythromycin current user & Amoxicillin current user & $1.63(1.32-2.03)$ & $1.98(1.52-2.56)$ \\
\hline \multicolumn{4}{|l|}{ Current vs. former use } \\
\hline Ciprofloxacin current user & Former user & $1.30(1.24-1.36)$ & $1.21(1.14-1.29)$ \\
\hline Levofloxacin current user & Former user & $1.63(1.56-1.70)$ & $1.43(1.35-1.52)$ \\
\hline Moxifloxacin current user & Former user & $1.35(1.13-1.60)$ & $1.36(1.09-1.69)$ \\
\hline Azithromycin current user & Former user & $1.22(1.16-1.28)$ & $1.21(1.13-1.28)$ \\
\hline Clarithromycin current user & Former user & $0.85(0.71-1.02)$ & $0.76(0.60-0.96)$ \\
\hline Erythromycin current user & Former user & $1.95(1.57-2.43)$ & $2.09(1.61-2.73)$ \\
\hline Amoxicillin current user & Former user & $1.24(1.19-1.29)$ & $1.14(1.08-1.21)$ \\
\hline Fluconazole current user & Former user & $1.58(1.53-1.65)$ & $1.45(1.38-1.52)$ \\
\hline Ondansetron current user & Former user & $1.77(1.72-1.83)$ & $1.83(1.76-1.90)$ \\
\hline \multicolumn{4}{|l|}{ Demographics } \\
\hline Female & Male & $0.70(0.69-0.70)$ & $0.70(0.69-0.71)$ \\
\hline Part D since 2008 & Part D since 2007 & $0.98(0.96-1.00)$ & $1.01(0.99-1.03)$ \\
\hline Part D since 2009 & Part D since 2007 & $0.98(0.96-1.00)$ & $0.91(0.89-0.94)$ \\
\hline Part D since 2010 & Part D since 2007 & $0.87(0.85-0.88)$ & $0.87(0.85-0.90)$ \\
\hline Part D since 2011 & Part D since 2007 & $0.84(0.82-0.85)$ & $0.82(0.80-0.85)$ \\
\hline Part D since 2012 & Part D since 2007 & $0.78(0.76-0.79)$ & $0.76(0.74-0.78)$ \\
\hline Part D since 2013 & Part D since 2007 & $0.67(0.66-0.69)$ & $0.64(0.62-0.66)$ \\
\hline Part D since 2014 & Part D since 2007 & $0.58(0.57-0.59)$ & $0.56(0.55-0.58)$ \\
\hline Part D since 2015 & Part D since 2007 & $0.59(0.57-0.61)$ & $0.58(0.56-0.60)$ \\
\hline African American & White & $1.13(1.11-1.14)$ & $1.02(1.00-1.05)$ \\
\hline Hispanic & White & $0.78(0.77-0.80)$ & $0.71(0.69-0.73)$ \\
\hline Asian & White & $0.81(0.78-0.84)$ & $0.73(0.70-0.77)$ \\
\hline Other & White & $0.96(0.93-0.99)$ & $0.97(0.93-1.01)$ \\
\hline Ever dual & Non dual no LIS & $1.04(1.03-1.06)$ & $0.98(0.96-1.00)$ \\
\hline Non-dual, LIS & Non dual no LIS & $1.45(1.42-1.48)$ & $1.10(1.07-1.14)$ \\
\hline Living in rural areas & No & $0.98(0.97-0.99)$ & $0.91(0.90-0.92)$ \\
\hline \multicolumn{4}{|l|}{ Proarrhythmic risk factors } \\
\hline Ischemic heart disease & No & $1.80(1.78-1.82)$ & $2.05(2.02-2.08)$ \\
\hline Hypothyroidism & No & $0.97(0.96-0.98)$ & $0.98(0.96-1.00)$ \\
\hline Chronic kidney disease & No & $1.35(1.33-1.36)$ & $1.19(1.17-1.21)$ \\
\hline Bradycardia & No & $1.53(1.52-1.55)$ & $1.82(1.79-1.85)$ \\
\hline $\begin{array}{l}\text { Electrolyte imbalance (including hypoka- } \\
\text { lemia) }\end{array}$ & No & $1.24(1.22-1.26)$ & $1.17(1.15-1.19)$ \\
\hline Heart failure & No & $2.44(2.41-2.47)$ & $2.75(2.71-2.80)$ \\
\hline Liver disease and cirrhosis & No & $1.15(1.13-1.17)$ & $1.13(1.11-1.16)$ \\
\hline Acute myocardial infarction & No & $1.19(1.17-1.22)$ & $1.17(1.14-1.21)$ \\
\hline
\end{tabular}


Table 2 (continued)
$V A$ ventricular arrhythmia, $S D$ sudden death, $H R$ hazard ratio, $C I$ confidence interval, LIS low-income subsidy

\subsection{Chronic-Usage Drugs}

Both SSRI antidepressants increased the risk for VA and SD. Current users of citalopram had increased risk of $14 \%$ (HR 1.14; 95\% CI 1.11-1.16) compared to former users, 6\% (HR 1.06; 95\% CI 1.03-1.08) compared to SNRI (our control antidepressant) current users, and 3\% (HR 1.03; 95\% CI 1.02-1.05) compared to never users (Table 3). The risk was generally higher with escitalopram. Current users of escitalopram had increased risk of $31 \%$ (HR 1.31 ; 95\% CI 1.28-1.35) compared to former users, $21 \%$ (HR $1.21 ; 95 \%$ CI 1.18-1.23) compared to SNRI current users, and 18\% (HR 1.18 ; 95\% CI 1.16-1.20) compared to never users. When current users were stratified according to the cumulative length of use, the risk was reduced for patients with cumulative use of 12 months or more. Compared to never users, the risk for current users of citalopram flipped from an increase of 23\% (HR 1.23; 95\% CI 1.20-1.26) in patients with less than 12 months of cumulative usage to a $14 \%$ (HR 0.86 ; 95\% CI 0.85-0.88) risk reduction for patients with cumulative use of over 12 months. The same pattern was observed when citalopram was compared to SNRI, with risk flipping from an increase of $27 \%$ (HR 1.27; 95\% CI 1.23-1.31) to a reduction of $12 \%$ (HR $0.88 ; 95 \%$ CI $0.85-0.90$ ) with prolonged use of both drugs. For escitalopram, compared to never users, the increased risk dropped from 34\% (HR 1.34; 95\% CI 1.31-1.37) to 4\% (HR 1.04; 95\% CI 1.01-1.06) with over 12 months of cumulative use. Compared to SNRIs, the increased risk of current users of escitalopram dropped from $39 \%$ (HR 1.39; 95\% CI 1.34-1.43) to 5\% (HR 1.05; 95\% CI $1.02-1.08)$ with prolonged use. Direct comparison of citalopram and escitalopram current users showed citalopram to be safer, with a reduction of risk of $13 \%$ (HR 0.87 ; 95\% CI $0.86-0.89$ ). The same trend was observed irrespective of the duration of cumulative use. As for SNRIs (the control drug for SSRI antidepressants), there was no consistent signal of increased or decreased risk. There was a minute reduction of risk of $2 \%$ (HR 0.98 ; 95\% CI 0.96-0.99) in current users compared to never users, which was not observed for patients with over 12 months of cumulative use. Compared to never users, current users of SNRIs with less than 12 months of cumulative use had a slight reduction of risk of $3 \%$ (HR 0.97; 95\% CI 0.94-0.99) for VA and SD, but an increase of risk of 3\% (HR 1.03; 95\% CI 1.00-1.06) for VA only.

For the antipsychotics, current users had an increased risk of $42 \%$ (HR 1.42 ; 95\% CI 1.39-1.45) compared to former users, and an increased risk of $118 \%$ (HR 2.18; 95\% CI 2.15-2.22) compared to never users. Similarly, for PDEIs, current users had an increased risk of 53\% (HR 1.53; 95\%
CI 1.51-1.55) compared to former users, and an increased risk of $156 \%$ (HR 2.56 ; 95\% CI 2.53-2.59) compared to never users. Similar to SSRI antidepressants, for both antipsychotics and PDEIs, the risks seemed to be mitigated with longer cumulative use. For hydroxychloroquine, there was an increased risk of $68 \%$ (HR 1.68; 95\% CI 1.66-1.71) when comparing current to never users, but no significant difference when compared with former users. For donepezil, there was an increased risk of $43 \%$ (HR 1.43; 95\% CI 1.41-1.45) when comparing current to never users, but there was a decreased risk of $13 \%$ (HR 0.87 ; 95\% CI $0.86-0.88$ ) when compared with former users.

Females had a $31 \%$ (HR 0.69; 95\% CI 0.68-0.70 reduction in risk, see Sect. 4). Younger patients (newer enrollment to Part D) had reduced risk. African Americans had an increased risk of 22\% (HR 1.22; 95\% CI 1.21-1.24) compared to Whites, while Hispanics and Asians had reduced risks of $23 \%$ (HR 0.77 ; 95\% CI $0.75-0.78$ ) and $45 \%$ (HR 0.55 ; 95\% CI $0.53-0.57$ ), respectively. Lower income was associated with increased risk, while rural residence was associated with decreased risk.

All the specific proarrhythmic risk factors were associated with increased risks. Among other co-morbidities, hypertension, anemia, and diabetes were associated with increased risks.

Results for the secondary outcome (VA alone) were mostly in agreement with the primary outcome, but with a smaller effect size. One notable exception was that for hydroxychloroquine current users compared to former users, there was a reduction in risk of $14 \%$ (HR 0.86; 95\% CI 0.84-0.88) for the secondary outcome while no difference was observed for the primary outcome. Electrolyte imbalance was associated with a slightly reduced risk for the secondary outcome. The sensitivity analysis without exclusion of patients with $<6$ months' follow-up showed no significant change in the results.

\section{Discussion}

Our study shows that CMS claims data can be a useful resource for detecting and monitoring serious arrhythmic events in QT-prolonging drugs. Among 17 drugs with known TdP risks, we are able to show increased risk in five of eight short-term drugs and all of nine chronic-use drugs. All control drugs (amoxicillin and SNRIs) were correctly identified as risk neutral. All the known patient factors for drug-induced TdP were correctly identified. In the US, Medicare is available to all citizens over 65 years of age, who are among the most vulnerable to QT prolongation 
Table 3 Results for chronic-usage drugs

\begin{tabular}{|c|c|c|c|}
\hline Treatment category & Reference group & $\begin{array}{l}\text { VA and/or SD } \\
\text { HR (95\% CI) }\end{array}$ & $\begin{array}{l}\text { VA } \\
\text { HR }(95 \% \text { CI })\end{array}$ \\
\hline \multicolumn{4}{|l|}{ Current vs. never user } \\
\hline Citalopram current user, all & Never user & $1.03(1.02-1.05)$ & $0.99(0.97-1.01)$ \\
\hline Citalopram current user, $\mathrm{CU} \leq 12 \mathrm{mo}$ & Never user & $1.23(1.20-1.26)$ & $1.05(1.02-1.09)$ \\
\hline Citalopram current user, $\mathrm{CU}>12 \mathrm{mo}$ & Never user & $0.86(0.85-0.88)$ & $0.93(0.90-0.95)$ \\
\hline Escitalopram current user, all & Never user & $1.18(1.16-1.20)$ & $1.16(1.14-1.19)$ \\
\hline Escitalopram current user, $\mathrm{CU} \leq 12 \mathrm{mo}$ & Never user & $1.34(1.31-1.37)$ & $1.26(1.22-1.30)$ \\
\hline Escitalopram current user, $\mathrm{CU}>12 \mathrm{mo}$ & Never user & $1.04(1.01-1.06)$ & $1.08(1.05-1.11)$ \\
\hline SNRI (control) current user, all & Never user & $0.98(0.96-0.99)$ & $1.02(1.00-1.04)$ \\
\hline SNRI (control) current user, $\mathrm{CU} \leq 12 \mathrm{mo}$ & Never user & $0.97(0.94-0.99)$ & $1.03(1.00-1.06)$ \\
\hline SNRI (control) current user, CU > $12 \mathrm{mo}$ & Never user & $0.99(0.97-1.00)$ & $1.01(0.98-1.03)$ \\
\hline Antipsychotic current user, all & Never user & $2.18(2.15-2.22)$ & $2.36(2.31-2.41)$ \\
\hline Antipsychotic current user, $\mathrm{CU} \leq 12 \mathrm{mo}$ & Never user & $2.62(2.57-2.68)$ & $3.53(3.44-3.62)$ \\
\hline Antipsychotic current user, CU > 12 mo & Never user & $1.81(1.77-1.86)$ & $1.57(1.52-1.63)$ \\
\hline PDEI current user, all & Never user & $2.56(2.53-2.59)$ & $2.97(2.93-3.01)$ \\
\hline PDEI current user, $\mathrm{CU} \leq 12 \mathrm{mo}$ & Never user & $3.16(3.11-3.20)$ & $3.90(3.83-3.96)$ \\
\hline PDEI current user, $\mathrm{CU}>12 \mathrm{mo}$ & Never user & $2.08(2.05-2.11)$ & $2.26(2.22-2.30)$ \\
\hline Hydroxychloroquine current user, all & Never user & $1.68(1.66-1.71)$ & $1.55(1.52-1.58)$ \\
\hline Hydroxychloroquine current user, $\mathrm{CU} \leq 12 \mathrm{mo}$ & Never user & $1.68(1.64-1.71)$ & $1.54(1.50-1.58)$ \\
\hline Hydroxychloroquine current user, $\mathrm{CU}>12 \mathrm{mo}$ & Never user & $1.69(1.67-1.72)$ & $1.56(1.53-1.59)$ \\
\hline Donepezil current user, all & Never user & $1.43(1.41-1.45)$ & $1.34(1.31-1.36)$ \\
\hline Donepezil current user, $\mathrm{CU} \leq 12 \mathrm{mo}$ & Never user & $1.59(1.57-1.62)$ & $1.30(1.27-1.33)$ \\
\hline Donepezil current user, CU > 12 mo & Never user & $1.29(1.27-1.31)$ & $1.38(1.34-1.41)$ \\
\hline \multicolumn{4}{|l|}{ Antidepressants comparison } \\
\hline Citalopram current user, all & Citalopram former user & $1.14(1.11-1.16)$ & $1.04(1.01-1.07)$ \\
\hline Escitalopram current user, all & Escitalopram former user & $1.31(1.28-1.35)$ & $1.26(1.22-1.30)$ \\
\hline Citalopram current user, all & Escitalopram current user, all & $0.87(0.86-0.89)$ & $0.85(0.83-0.88)$ \\
\hline Citalopram current user, $\mathrm{CU} \leq 12 \mathrm{mo}$ & Escitalopram current user, $\mathrm{CU} \leq 12 \mathrm{mo}$ & $0.92(0.89-0.95)$ & $0.84(0.80-0.88)$ \\
\hline Citalopram current user, $\mathrm{CU}>12 \mathrm{mo}$ & Escitalopram current user, $\mathrm{CU}>12 \mathrm{mo}$ & $0.83(0.81-0.86)$ & $0.86(0.83-0.90)$ \\
\hline Citalopram current user, all & SNRI current user. all & $1.06(1.03-1.08)$ & $0.97(0.95-1.00)$ \\
\hline Citalopram current user, $\mathrm{CU} \leq 12 \mathrm{mo}$ & SNRI current user, $\mathrm{CU} \leq 12 \mathrm{mo}$ & $1.27(1.23-1.31)$ & $1.03(0.98-1.07)$ \\
\hline Citalopram current user, $\mathrm{CU}>12 \mathrm{mo}$ & SNRI current user, $\mathrm{CU}>12 \mathrm{mo}$ & $0.88(0.85-0.90)$ & $0.92(0.89-0.95)$ \\
\hline Escitalopram current user, all & SNRI current user, all & $1.21(1.18-1.23)$ & $1.14(1.11-1.18)$ \\
\hline Escitalopram current user, $\mathrm{CU} \leq 12 \mathrm{mo}$ & SNRI current user, $\mathrm{CU} \leq 12 \mathrm{mo}$ & $1.39(1.34-1.43)$ & $1.22(1.17-1.27)$ \\
\hline Escitalopram current user, $\mathrm{CU}>12 \mathrm{mo}$ & SNRI current user, $\mathrm{CU}>12 \mathrm{mo}$ & $1.05(1.02-1.08)$ & $1.07(1.03-1.11)$ \\
\hline \multicolumn{4}{|l|}{ Current vs. former use of other drugs } \\
\hline Antipsychotic current user, all & Former user & $1.42(1.39-1.45)$ & $2.19(2.13-2.25)$ \\
\hline PDEI current user, all & Former user & $1.53(1.51-1.55)$ & $12.08(2.05-2.12)$ \\
\hline Hydroxychloroquine current user, all & Former user & $0.99(0.97-1.00)$ & $0.86(0.84-0.88)$ \\
\hline Donepezil current user, all & Former user & $0.87(0.86-0.88)$ & $0.92(0.90-0.94)$ \\
\hline \multicolumn{4}{|l|}{ Demographics } \\
\hline Female & Male & $0.69(0.68-0.70)$ & $0.70(0.69-0.70)$ \\
\hline Part D since 2008 & Part D since 2007 & $0.93(0.92-0.95)$ & $0.81(0.79-0.82)$ \\
\hline Part D since 2009 & Part D since 2007 & $0.93(0.91-0.94)$ & $0.85(0.84-0.87)$ \\
\hline Part D since 2010 & Part D since 2007 & $1.01(1.00-1.03)$ & $0.94(0.92-0.95)$ \\
\hline Part D since 2011 & Part D since 2007 & $0.92(0.91-0.94)$ & $0.68(0.67-0.70)$ \\
\hline Part D since 2012 & Part D since 2007 & $0.73(0.72-0.74)$ & $0.58(0.57-0.59)$ \\
\hline Part D since 2013 & Part D since 2007 & $0.99(0.97-1.00)$ & $0.87(0.85-0.89)$ \\
\hline Part D since 2014 & Part D since 2007 & $0.63(0.62-0.64)$ & $0.48(0.47-0.49)$ \\
\hline Part D since 2015 & Part D since 2007 & $0.55(0.54-0.56)$ & $0.50(0.49-0.52)$ \\
\hline
\end{tabular}


Table 3 (continued)

\begin{tabular}{llll}
\hline Treatment category & Reference group & VA and/or SD & $\begin{array}{c}\text { VA } \\
\text { HR (95\% CI) }\end{array}$ \\
\hline African American & & HR CI $)$ & $1.22(1.21-1.24)$ \\
Hispanic & White & $0.77(0.75-0.78)$ & $0.71(0.69-0.72)$ \\
Asian & White & $0.55(0.53-0.57)$ & $0.37(0.35-0.39)$ \\
Other & White & $1.61(1.58-1.64)$ & $1.15(1.12-1.18)$ \\
Ever dual & White & $1.03(1.02-1.04)$ & $1.04(1.02-1.05)$ \\
Non-dual, LIS & Non-dual no LIS & $1.60(1.57-1.62)$ & $1.26(1.23-1.28)$ \\
Living in rural areas & Non-dual no LIS & $0.92(0.91-0.93)$ & $0.77(0.76-0.78)$ \\
Proarrhythmic risk factors & No & $1.54(1.53-1.55)$ & $1.75(1.73-1.77)$ \\
Ischemic heart disease & & $1.03(1.02-1.04)$ & $1.02(1.01-1.03)$ \\
Hypothyroidism & No & $1.52(1.51-1.53)$ & $1.45(1.44-1.47)$ \\
Chronic kidney disease & No & $1.50(1.48-1.51)$ & $1.81(1.79-1.83)$ \\
Bradycardia & No & $1.07(1.06-1.08)$ & $0.97(0.96-0.98)$ \\
Electrolyte imbalance (including hypokalemia) & No & $2.84(2.82-2.87)$ & $3.21(3.18-3.25)$ \\
Heart failure & No & $1.48(1.46-1.49)$ & $1.61(1.59-1.63)$ \\
Liver disease and cirrhosis & No & $1.31(1.29-1.33)$ & $1.27(1.25-1.29)$ \\
Acute myocardial infarction & No & & \\
\hline
\end{tabular}

$V A$ ventricular arrhythmia, $S D$ sudden death, $H R$ hazard ratio, $C I$ confidence interval, $C U$ cumulative use, mo months, $L I S$ low-income subsidy, $S N R I$ serotonin-norepinephrine reuptake inhibitor, PDEI phosphodiesterase inhibitor

and its deadly consequences. Medicare beneficiaries, once enrolled, generally stay within the system, making longitudinal follow-up possible to detect rare events. It is estimated that $74 \%$ of Medicare beneficiaries are enrolled in Part D [34]. Our study included over 1.2 million patients with over 5 million patient-years of follow-up, which is among the largest of its kind.

Among the fluoroquinolones, our findings suggest that ciprofloxacin is the safest. Levofloxacin and moxifloxacin are both associated with increased risk of VA and/or SD in all comparisons (former users, never users, and amoxicillin controls). This is in line with the finding that both drugs cause QT prolongation in humans to a similar degree, more so than ciprofloxacin [22]. Ciprofloxacin is the weakest hERG (human ether-a-go-go-related gene) potassium channel blocker among the fluoroquinolones, which is considered the underlying mechanism for QT prolongation. In a large retrospective study, levofloxacin was found to be associated with significant increase in cardiovascular mortality and cardiac arrhythmia [35]. Both levofloxacin and moxifloxacin carry specific warnings about QT prolongation and VA in their product labels. In our study, ciprofloxacin is similar to amoxicillin in that there is only increased risk compared to former users, which could be an indication bias since antibiotics are prescribed for acute infective diseases that could increase the likelihood of adverse outcomes.

Among the macrolides, we did not find increased risk with azithromycin and clarithromycin. Erythromycin is consistently associated with increased risk in our study.
Multiple studies have placed erythromycin as the highest risk for cardiac arrhythmias among the macrolides [36-39]. Between azithromycin and clarithromycin, it is not clear which is safer. Other studies show conflicting results [37-42]. In our study, clarithromycin has even less risk than amoxicillin, which can be due to indication bias. Clarithromycin is the only macrolide indicated as combination therapy for eradication of Helicobacter pylori in patients with peptic ulcer diseases who do not have an acute infection like patients on other antibiotics.

As for the two non-antibiotic short-term drugs, fluconazole and ondansetron, our results show strong signals for their increased risks of $123-205 \%$ and $58-77 \%$ when compared to never and former users, respectively.

Among the chronic-usage drugs, we show that both SSRI antidepressants, citalopram and escitalopram, have increased risks in all comparisons-never users, former users, and SNRI controls. Escitalopram is associated with higher risk than citalopram. This is in agreement with two meta-analyses [43, 44]. Our findings are not in support of the appeal to favor escitalopram over citalopram based on the smaller degree of QT prolongation on therapeutically equivalent doses [45]. However, according to the drug labels, QT prolongation and TdP are specifically mentioned as warnings only for citalopram and not escitalopram. As for antipsychotics, our results show increased risk in comparison with former and never users, which is consistent with other studies [23]. 
Table 4 Effect of sex on outcome

\begin{tabular}{lll}
\hline & Female & Male \\
\hline $\begin{array}{l}\text { Ventricular arrhythmia and/or sudden } \\
\text { death }\end{array}$ & & \\
Overall & $2.33 \%$ & \\
On any study drug & $2.47 \%$ & $4.12 \%$ \\
Not on study drug & $1.92 \%$ & $4.24 \%$ \\
OR & $1.30(95 \%$ CI $1.25-1.34)$ & $3.29 \%$ \\
Ventricular arrhythmia & & 1.10 (95\% CI 1.07-1.13) \\
Overall & $1.58 \%$ & \\
On any study drug & $1.65 \%$ & $2.91 \%$ \\
Not on study drug & $1.36 \%$ & $2.98 \%$ \\
OR & $1.22(95 \%$ CI 1.16-1.27) & $2.78 \%$ \\
\end{tabular}

$O R$ odds ratio, $C I$ confidence interval
Another interesting finding in our study is the effect of length of use of chronic drugs on risk levels. In all the chronic-usage drugs that are shown to have increased risks, the effect is lower in patients who have cumulative use of over 12 months. This shows that there is no evidence for cumulative effect in prolonged use. Several studies have shown that arrhythmogenic risks for long-term drugs are higher when the drugs are first started compared to steady users $[23,46]$. It is unclear whether and how patients become less vulnerable to the cardiac side effects of the drugs on prolonged use. It is possible that patients who suffer from severe side effects early in their treatment have stopped taking the drug and never become prolonged users. Immortal time bias can also be a factor since patients have to live for 12 months to qualify as a prolonged user.

Although drug-induced TdP is sometimes regarded as an idiosyncratic event, females are considered to be more susceptible [47]. This apparently contradicts our findings. However, this has to be interpreted with care. The events we are observing, VA and SD, can have other causes besides druginduced arrhythmia. Many of the non-drug-related causes (e.g., coronary heart disease) are more common in males. In our study, both VA and SD are overall more common in males, as shown in Table 4. This explains the overall lower hazard ratios for females in our regression models. However, for patients taking any of the study drugs, the odds ratios (ORs) for VA and SD [OR for female 1.30 (CI 1.25-1.34) vs. male 1.10 (CI 1.07-1.13)] and VA [OR for female 1.22 (CI 1.16-1.27) vs. male 1.07 (CI 1.04-1.11)] are significantly higher for females. This suggests that being female increases the risk of drug-induced arrhythmia.

Some known co-morbidities that increase the risk for drug-induced TdP are confirmed in our study. They include ischemic heart disease, electrolyte imbalance and hypokalemia, bradycardia, acute myocardial infarction, heart failure, and chronic kidney and liver disease.
As with other observational studies based on claims data, misclassification errors are a potential problem. Our study relies on ICD codes to identify the outcome events of ventricular arrhythmias and sudden death. This is not a foolproof method, but is a common method and has been shown to give a reasonably high positive predictive value of $85 \%$ (95\% CI 78-91) for identifying ventricular arrhythmias and sudden death not due to extrinsic (e.g., traumatic) causes [23, 48].

We recognize the following additional limitations. We have used propensity score weighting to compensate for potential bias, but there could be other unadjusted confounders. The outcome events we studied, VA and SD, are heterogeneous conditions of which drug-induced $\mathrm{TdP}$ is only one of the possible causes. There are other factors that affect the risk of QT-prolonging drugs, such as dosage and drug-drug interactions, which we have not covered in our study.

\section{Conclusion}

Medicare data can be utilized to perform post-marketing surveillance and monitoring of the proarrhythmic risk of QT-prolonging drugs. We are able to detect increased risk for ventricular arrhythmia and sudden death in 14 out of 17 drugs with known TdP risk, as distinct from control drugs that are not associated with increased risk. Ciprofloxacin is the safest fluoroquinolone. Both azithromycin and clarithromycin are safe among the macrolides. Escitalopram is associated with higher risk than citalopram, and both drugs have increased risk compared to SNRIs.

Supplementary Information The online version contains supplementary material available at https://doi.org/10.1007/s40801-021-00230-1. 
Acknowledgements The authors would like to thank Seo Baik for assisting in the statistical analysis and revision of the manuscript.

\section{Declarations}

Funding This research was supported in part by the Intramural Research Program of the National Institutes of Health, National Library of Medicine.

Conflict of Interest The authors declare that they have no competing interests.

Availability of Data and Material The data source used in this study can be accessed through the CMS Virtual Research Data Center.

Ethics Approval This study only involved access to de-identified patient data, and was rated as exempt from human subject research review by the Office of Human Subjects Research Protections, National Institutes of Health.

Author Contributions KWF and CJM contributed to the study conception and design. Data collection and analysis was performed by FB. All authors participated in the discussion and interpretation of the results. The first draft of the manuscript was written by KWF. All authors contributed to revision of the manuscript. All authors read and approved the final manuscript.

Open Access This article is licensed under a Creative Commons Attribution-NonCommercial 4.0 International License, which permits any non-commercial use, sharing, adaptation, distribution and reproduction in any medium or format, as long as you give appropriate credit to the original author(s) and the source, provide a link to the Creative Commons licence, and indicate if changes were made. The images or other third party material in this article are included in the article's Creative Commons licence, unless indicated otherwise in a credit line to the material. If material is not included in the article's Creative Commons licence and your intended use is not permitted by statutory regulation or exceeds the permitted use, you will need to obtain permission directly from the copyright holder. To view a copy of this licence, visit http://creativecommons.org/licenses/by-nc/4.0/.

\section{References}

1. Clark-Kennedy AE. Discussion on the action of quinidine in cases of cardiac disease. Proc R Soc Med. 1923;16(Ther Pharmacol Sect):32-8

2. Dessertenne F. Ventricular tachycardia with 2 variable opposing foci. Arch Mal Coeur Vaiss. 1966;59(2):263-72.

3. Roden DM. Drug-induced prolongation of the QT interval. N Engl J Med. 2004;350(10):1013-22. https://doi.org/10.1056/NEJMr a032426.

4. U.S. Food and Drug Administration. E14 Clinical evaluation of QT/QTc interval prolongation and proarrhythmic potential for non-antiarrhythmic drugs - questions and answers (R3) guidance for industry. 2017. https://www.fda.gov/media/71379/download. Accessed 29 Jan 2021.

5. Shah RR. Drugs, QTc interval prolongation and final ICH E14 guideline: an important milestone with challenges ahead. Drug Saf. 2005;28(11):1009-28. https://doi.org/10.2165/00002018200528110-00003.
6. Ninan B, Wertheimer A. Withdrawing drugs in the U.S. versus other countries. Inov Pharm. 2012;3:1-12.

7. Roden DM. Predicting drug-induced QT prolongation and torsades de pointes. J Physiol. 2016;594(9):2459-68. https://doi. org/10.1113/JP270526.

8. Roden DM, Harrington RA, Poppas A, Russo AM. Considerations for Drug Interactions on QTc in Exploratory COVID-19 (Coronavirus Disease 2019) Treatment, American Heart Association Media Alerts. 2020. https://www.ahajournals.org/doi/pdf/10.1161/ CIRCULATIONAHA.120.047521. Accessed 29 Jan 2021.

9. FDA Drug Safety Communication [4-24-2020]. FDA cautions against use of hydroxychloroquine or chloroquine for COVID19 outside of the hospital setting or a clinical trial due to risk of heart rhythm problems. 2020. https://www.fda.gov/drugs/drugsafety-and-availability/fda-cautions-against-use-hydroxychloroqu ine-or-chloroquine-covid-19-outside-hospital-setting-or. Accessed 29 Jan 2021.

10. Coronavirus (COVID-19) Update [6-15-2020]: FDA Revokes Emergency Use Authorization for Chloroquine and Hydroxychloroquine. 2020. https://www.fda.gov/news-events/press -announcements/coronavirus-covid-19-update-fda-revokesemergency-use-authorization-chloroquine-and\#: :text=Today $\% 2$ C\%20the\%20U.S.\%20Food $\% 20$ and,clinical $\% 20$ trial $\% 20$ was \%20unavailable\%2C\%20or. Accessed 29 Jan 2021.

11. Boulware DR, Pullen MF, Bangdiwala AS, Pastick KA, Lofgren $\mathrm{SM}$, Okafor EC, et al. A randomized trial of hydroxychloroquine as postexposure prophylaxis for Covid-19. N Engl J Med. 2020. https://doi.org/10.1056/NEJMoa2016638.

12. Mercuro NJ, Yen CF, Shim DJ, Maher TR, Mccoy CM, Zimetbaum PJ, et al. Risk of QT interval prolongation associated with use of hydroxychloroquine with or without concomitant azithromycin among hospitalized patients testing positive for coronavirus disease 2019 COVID-19. JAMA Cardiol. 2019. https://doi.org/10.1001/jamacardio.2020.1834.

13. Cavalcanti AB, Zampieri FG, Rosa RG, Azevedo LCP, Veiga VC, Avezum A, et al. Hydroxychloroquine with or without azithromycin in mild-to-moderate Covid-19. N Engl J Med. 2020. https://doi.org/10.1056/NEJMoa2019014.

14. Hondeghem LM. QT prolongation is an unreliable predictor of ventricular arrhythmia. Heart Rhythm. 2008;5(8):1210-2. https ://doi.org/10.1016/j.hrthm.2008.05.006.

15. Schwartz PJ, Woosley RL. Predicting the unpredictable: druginduced QT prolongation and Torsades de Pointes. J Am Coll Cardiol. 2016;67(13):1639-50. https://doi.org/10.1016/j. jacc.2015.12.063.

16. Lester RM, Paglialunga S, Johnson IA. QT Assessment in early drug development: the long and the short of It. Int J Mol Sci. 2019. https://doi.org/10.3390/ijms20061324.

17. Slama TG. Minimizing the risk for QT interval prolongation. $\mathbf{J}$ Family Pract. 2005;54(6):S15-7.

18. Heist EK, Ruskin JN. Drug-induced arrhythmia. Circulation. 2010;122(14):1426-35. https://doi.org/10.1161/CIRCULATIO NAHA.109.894725.

19. Kramer DB, Zimetbaum PJ. Long-QT syndrome. Cardiol Rev. 2011;19(5):217-25. https://doi.org/10.1097/CRD.0b013e3182 203504.

20. Letsas K, Tsikrikas S, Letsas G, Sideris A. Drug-induced proarrhythmia: QT interval prolongation and torsades de pointes. Hosp Chron. 2011;6:118-22.

21. Tisdale JE, Jaynes HA, Kingery JR, Mourad NA, Trujillo TN, Overholser BR, et al. Development and validation of a risk score to predict QT interval prolongation in hospitalized patients. Circ Cardiovasc Qual Outcomes. 2013;6(4):479-87. https:// doi.org/10.1161/CIRCOUTCOMES.113.000152.

22. Owens RC Jr. Risk assessment for antimicrobial agentinduced QTc interval prolongation and torsades de pointes. 
Pharmacotherapy. 2001;21(3):301-19. https://doi.org/10.1592/ phco.21.3.301.34206.

23. Leonard CE, Freeman CP, Newcomb CW, Bilker WB, Kimmel SE, Strom BL, et al. Antipsychotics and the risks of sudden cardiac death and all-cause death: cohort studies in medicaid and dually-eligible medicaid-medicare beneficiaries of five states. J Clin Exp Cardiolog. 2013;10(6):1-9. https://doi. org/10.4172/2155-9880.S10-006.

24. Sarganas G, Garbe E, Klimpel A, Hering RC, Bronder E, Haverkamp W. Epidemiology of symptomatic drug-induced long QT syndrome and Torsade de Pointes in Germany. Europace. 2014;16(1):101-8. https://doi.org/10.1093/europace/ eut214.

25. Centers for Medicare and Medicaid Services CMS. CMS Announces New Data Sharing Tool. 2013. https://www.cms. gov/newsroom/press-releases/cms-announces-new-data-shari ng-tool. Accessed 29 Jan 2021.

26. Research Data Assistance Center (ResDAC). Strengths and Limitations of CMS Administrative Data in Research. 2018. https:// www.resdac.org/articles/strengths-and-limitations-cms-administra tive-data-research. Accessed 29 Jan 2021.

27. Administration on Aging USDoHaHS. 2018 Profile of Older Americans. 2018. https://acl.gov/aging-and-disability-in-ameri $\mathrm{ca} /$ data-and-research/profile-older-americans. Accessed 29 Jan 2021.

28. Putter H, Fiocco M, Geskus RB. Tutorial in biostatistics: competing risks and multi-state models. Stat Med. 2007;26(11):2389430. https://doi.org/10.1002/sim.2712.

29. CredibleMeds: A Trusted Partner Providing Reliable Information on Medicines. 2020. https://www.crediblemeds.org/. Accessed 29 Jan 2021

30. Stricker BH, Stijnen T. Analysis of individual drug use as a timevarying determinant of exposure in prospective population-based cohort studies. Eur J Epidemiol. 2010;25(4):245-51. https://doi. org/10.1007/s10654-010-9451-7.

31. Shintani AK, Girard TD, Eden SK, Arbogast PG, Moons KG, Ely EW. Immortal time bias in critical care research: application of time-varying Cox regression for observational cohort studies. Crit Care Med. 2009;37(11):2939-45. https://doi.org/10.1097/ CCM.0b013e3181b7fbbb.

32. Cadarette SM, Gagne JJ, Solomon DH, Katz JN, Sturmer T. Confounder summary scores when comparing the effects of multiple drug exposures. Pharmacoepidemiol Drug Saf. 2010;19(1):2-9. https://doi.org/10.1002/pds.1845.

33. Austin PC. The use of propensity score methods with survival or time-to-event outcomes: reporting measures of effect similar to those used in randomized experiments. Stat Med. 2014;33(7):1242-58. https://doi.org/10.1002/sim.5984.

34. Centers for Medicare \& Medicaid Services CCDW. Medicare Part D Charts. 2020. https://www2.ccwdata.org/web/guest/medicarecharts/medicare-part-d-charts.

35. Rao GA, Mann JR, Shoaibi A, Bennett CL, Nahhas G, Sutton SS, et al. Azithromycin and levofloxacin use and increased risk of cardiac arrhythmia and death. Ann Fam Med. 2014;12(2):121-7. https://doi.org/10.1370/afm.1601.
36. Ray WA, Murray KT, Meredith S, Narasimhulu SS, Hall K, Stein $\mathrm{CM}$. Oral erythromycin and the risk of sudden death from cardiac causes. N Engl J Med. 2004;351(11):1089-96. https://doi. org/10.1056/NEJMoa040582.

37. Shaffer D, Singer S, Korvick J, Honig P. Concomitant risk factors in reports of torsades de pointes associated with macrolide use: review of the United States Food and Drug Administration Adverse Event Reporting System. Clin Infect Dis. 2002;35(2):197-200. https://doi.org/10.1086/340861.

38. Milberg P, Eckardt L, Bruns HJ, Biertz J, Ramtin S, Reinsch N, et al. Divergent proarrhythmic potential of macrolide antibiotics despite similar QT prolongation: fast phase 3 repolarization prevents early afterdepolarizations and torsade de pointes. J Pharmacol Exp Ther. 2002;303(1):218-25. https://doi.org/10.1124/ jpet.102.037911.

39. Cheng YJ, Nie XY, Chen XM, Lin XX, Tang K, Zeng WT, et al. The role of macrolide antibiotics in increasing cardiovascular risk. J Am Coll Cardiol. 2015;66(20):2173-84. https://doi. org/10.1016/j.jacc.2015.09.029.

40. Giudicessi JR, Ackerman MJ. Azithromycin and risk of sudden cardiac death: guilty as charged or falsely accused? Clevel Clin J Med. 2013;80(9):539-44. https://doi.org/10.3949/ccjm.80a.13077

41. Lu ZK, Yuan J, Li M, Sutton SS, Rao GA, Jacob S, et al. Cardiac risks associated with antibiotics: azithromycin and levofloxacin. Expert Opin Drug Saf. 2015;14(2):295-303. https://doi. org/10.1517/14740338.2015.989210.

42. Zaroff J, Cheetham C, Palmetto N. Association of azithromycin use with cardiovascular mortality. JAMA Netw Open. 2020;3(6):e208199. https://doi.org/10.1001/jamanetworkopen .2020 .8199 .

43. Beach SR, Kostis WJ, Celano CM, Januzzi JL, Ruskin JN, Noseworthy PA, et al. Meta-analysis of selective serotonin reuptake inhibitor-associated QTc prolongation. J Clin Psychiatry. 2014;75(5):e441-9. https://doi.org/10.4088/JCP.13r08672.

44. Funk KA, Bostwick JR. A comparison of the risk of QT prolongation among SSRIs. Ann Pharmacother. 2013;47(10):1330-41. https://doi.org/10.1177/1060028013501994.

45. Keller DL. Prescribe escitalopram instead of citalopram. Am J Med. 2013;126(6):e21. https://doi.org/10.1016/j.amjme d.2012.10.024.

46. Straus SM, Sturkenboom MC, Bleumink GS, Dieleman JP, van der Lei J, de Graeff PA, et al. Non-cardiac QTc-prolonging drugs and the risk of sudden cardiac death. Eur Heart J. 2005;26(19):200712. https://doi.org/10.1093/eurheartj/ehi312.

47. Drew BJ, Ackerman MJ, Funk M, Gibler WB, Kligfield P, Menon $\mathrm{V}$, et al. Prevention of torsade de pointes in hospital settings: a scientific statement from the American Heart Association and the American College of Cardiology Foundation. J Am Coll Cardiol. 2010;55(9):934-47. https://doi.org/10.1016/j.jacc.2010.01.001.

48. Hennessy S, Leonard CE, Freeman CP, Deo R, Newcomb C, Kimmel SE, et al. Validation of diagnostic codes for outpatientoriginating sudden cardiac death and ventricular arrhythmia in Medicaid and Medicare claims data. Pharmacoepidemiol Drug Saf. 2010;19(6):555-62. https://doi.org/10.1002/pds.1869. 